\title{
Prevention of Murine Acute Graft-versus-Host Disease by Recipient-Derived Paired Immunoglobulin-Like Receptor B Lentivirus-Transfected Dendritic Cells
}

\author{
Juan Zhao a,b Yi Luo ${ }^{a}$ Xiaochong Wanga,c Hao Zhou ${ }^{a}$ Qiubai Lia Yong You ${ }^{a}$ \\ Ping Zou ${ }^{a}$ \\ a Institute of Hematology, Union Hospital, Tongji Medical College, Huazhong University of Science and Technology, \\ and ${ }^{b}$ Department of Hematology, Wuhan Center Hospital, Wuhan, and 'Department of Oncology, Suizhou Center \\ Hospital, Suizhou, People's Republic of China
}

\section{Key Words}

Allogeneic stem cells $\cdot$ Bone marrow transplantation •

Dendritic cells $\cdot$ Graft-versus-host disease $\cdot$ Paired

immunoglobulin-like receptor $B$

\begin{abstract}
Direct interaction between T-suppressor and dendritic cells (DCs) results in DC tolerance by inducing upregulation of immunoglobulin-like transcript (ILT) 3 and ILT4. DCs were treated with a lentiviral vector containing paired immunoglobulin-like B gene (PIR-B) DCs and the effect of PIR-B-DCs on graft-versus-host disease (GVHD) was analyzed after allogeneic bone marrow (BM) transplantation (BMT). Therefore, $1 \times 10^{6}$ recipient-derived PIR-B-DCs were injected into $B A L B / C\left(H-2 k^{d}\right)$ mice using BM-splenocyte grafts from major histocompatibility complex-disparate C57BL/6 $\left(\mathrm{H}-2 \mathrm{k}^{\mathrm{b}}\right)$. Our results showed that PIR-B-DCs deficient in surface costimulatory molecules had higher PIR-B protein expression than immature DCs and interleukin 10-treated DCs. Survival analysis showed PIR-B-DC cotransplantation resulted in significant prolongation of allograft survival (mean survival time: 46.0 \pm 13.6 vs. $17.4 \pm 3.6$ days in untreated MST; $p<0.01$ ). Furthermore, samples from the liver and skin of a mouse did not
\end{abstract}

show clinical or histological signs of GVHD following the injection of PIR-B-DCs. These results demonstrated that PIR-BDC cotransplantation may attenuate the severity of GVHD after BMT.

Copyright $\odot 2010$ S. Karger AG, Basel

\section{Introduction}

Bone marrow (BM) transplantation (BMT) is an established therapy for the treatment of many hematologic malignancies. However, the clinical application of this therapy is limited to patients with a closely matched donor. Allogeneic BMT across multiple major histocompatibility (MHC) disparities is characterized by high morbidity and mortality associated with severe acute graftversus-host disease (aGVHD). The severity of these complications has prevented the clinical application of allogeneic BMT. Therefore, the aim of therapy is to eliminate GVHD without affecting tumor response.

Dendritic cells (DCs) are the most potent antigen-presenting cells (APCs) that induce and regulate immune reactivity [1]. Mature DCs can stimulate T cells through high expression of MHCII and costimulatory molecules 
(CMs), while immature DCs (imDCs) inhibit T-cell responses and induce tolerance. imDCs become tolerogenic to inhibit the activation of T-helper cells $\left(\mathrm{T}_{\mathrm{H}}\right)$ when they interact with antigen-specific CD8+CD28- T-suppressor $\left(\mathrm{T}_{S}\right)$ cells [2]. High expression of immunoglobulin-like transcript (ILT) 3 and ILT4 is a general feature of tolerogenic DCs [3]. Tolerogenic DCs with increased ILT3 and ILT4 expression induce $\mathrm{T}_{\mathrm{H}}$ anergy inhibiting the capacity of alloreactive $\mathrm{CD} 4+\mathrm{T}$ cells to proliferate [2]. Meanwhile, tolerogenic DCs stimulate the generation of T-regulatory $\left(\mathrm{T}_{\mathrm{R}}\right)$ and $\mathrm{T}_{\mathrm{S}}$ cells, which in turn continue the cascade of suppression by tolerizing other DCs [3]. Circulating tolerogenic DCs and T cells suppressing the immune response abate the symptoms of GVHD and preserve allograft function.

Paired immunoglobulin-like receptor B (PIR-B), expressed on APCs of rodents, comprise a MHC class I recognition system. Recently, a report showed that the molecular structure and function of PIR-B resembles ILT4, which is expressed in humans [4]. PIR-B bears a long cytoplasmic domain that contains three potential immunoreceptor tyrosine-based inhibitory motifs and inhibits the receptor-mediated activation signal. After transfer of allogeneic splenocytes into PIR-B-deficient mice, the mice showed exacerbated GVHD, which was due to augmented activation of recipient DCs [5]. In this study, we evaluated the effects of PIR-B lentivirus on murine BMderived DC maturation and function compared with imDCs and IL-10-treated DCs (IL-10-DCs), including effects on morphology and expression of MHCII, CMs and PIR-B, and used a murine aGVHD model as an additional in vivo assay for suppressor function in a nonautoimmune disease-prone strain.

\section{Materials and Methods}

\section{Mice and Lentivirus}

$\mathrm{BALB} / \mathrm{c}\left(\mathrm{H}-2 \mathrm{k}^{\mathrm{d}}\right)$ mice were purchased from the Vital River Laboratories (China). C57BL/6 $\left(\mathrm{H}-2 \mathrm{k}^{\mathrm{b}}\right)$ mice were obtained from the breeding facility of the Organ Transplantation Department at TongJi Hospital, Huazhong University of Science and Technology. Donors were male and between 6 and 12 weeks of age; recipients were female and at least 8 weeks of age. All mice were maintained in specific pathogen-free conditions and in accordance with institutional guidelines. PIR-B lentiviruses were constructed as described previously [6].

\section{Generation of BM-Derived DCs and Lentivirus Infection of} Murine DCs

DCs were generated from BM as described previously [7]. Briefly, imDCs were prepared by culturing BM cells obtained from $\mathrm{BALB} / \mathrm{c}$ mice with granulocyte-macrophage colony-stimu- lating factor (GM-CSF, $20 \mathrm{ng} / \mathrm{ml}$; Peprotech, USA). IL-10-DCs were generated from $\mathrm{BM}$ cells obtained from $\mathrm{BALB} / \mathrm{c}$ mice and cultured with GM-CSF (20 ng/ml) and recombinant murine IL-10 ( $20 \mathrm{ng} / \mathrm{ml}$; Peprotech). On day 5, non-adherent cells were removed and fresh medium containing cytokine was added. On day 6 or 7 of culture, nonadherent cells and loosely adherent DC aggregates were harvested for the experiments.

Cells were then infected with PIR-B lentivirus at a multiplicity of infection of 5 for $6 \mathrm{~h}$. Subsequently, the lentivirus-containing medium was removed and substituted by DC culture medium. The infected cells were left to express the transgenes of interest for another 4 and 5 days before further use.

\section{Antibodies and Flow Cytometry}

Expression of PIR-B, CD80, CD86, CD40 and MHCII on imDCs, IL-10-DCs and PIR-B lentivirus-transfected DCs was determined as described previously [8]. Four hundred microliters of cells at a concentration of $1 \times 10^{5} / \mathrm{ml}$ were incubated with FITC-and PE-labeled monoclonal antibodies (Biolegend, USA) at the optimal concentration for immunostaining for $45 \mathrm{~min}$ at $4^{\circ} \mathrm{C}$. Cells were washed and resuspended in $400 \mu \mathrm{l}$ PBS, then analyzed on a FACScalibur flow cytometer (BD, USA). We used isotypematched antibodies as controls to avoid nonspecific binding to $\mathrm{Fc}$ receptors.

Western Blots and Reverse Transcription-Polymerase Chain Reaction

Western blot analysis was carried out using $40 \mu \mathrm{g}$ of cell lysates. The primary antibody was added in 5\% (v/v) milk. Proteins were visualized using ECL.

Total RNA that was obtained from cells reverse-transcribed (RT) into cDNA, and then polymerase chain reaction (PCR) amplification of cDNA was performed using a thermal cycler. The sequences of the PCR primers used in this study were as follows: PIR-B forward, CCTGTGGAGCTCACAGTCTCAG; PIR-B reverse, GTGTTCAGTTGTTCCCTTGACATGA; $\beta$-actin forward, CTGGCACCACACCTCCTACA; $\beta$-actin reverse, ATGACTTGCGCACAGGAGGA, and mouse Sry forward, CTAAGCCATGTACCACCT, and Sry reverse, CTCTCTCTTTATCAATTTTTTCT. The primers were synthesized by Shanghai Invitrogen Biological Engineering. After amplification, the products were separated on $1.5 \%(\mathrm{w} / \mathrm{v})$ agarose gels and stained with ethidium bromide.

\section{aGVHD Model}

aGVHD was induced as described previously [9]. In brief, $\mathrm{BALB} / \mathrm{c}$ hosts were lethally irradiated $(8.5 \mathrm{~Gy})$ and injected intravenously within $6 \mathrm{~h}$ with $1 \times 10^{7} \mathrm{BM}$ cells and $2 \times 10^{7}$ spleen mononuclear cells from C57BL/6 mice. Recipients were divided into four groups and received intravenous injections of various additional DCs from BALB/c mice at the time of BM-splenocyte transplantation. The imDCs group $(\mathrm{n}=15)$ received $1 \times 10^{6}$ imDCs; the IL-10-DC group $(n=15) 1 \times 10^{6}$ IL-10-DCs; the PIRB-DCs group $(\mathrm{n}=15) 1 \times 10^{6}$ PIR-B lentivirus-transfected DCs, and the BMT group $(\mathrm{n}=10)$ received no DCs. The day of transplantation was designated as day 0 . For survival studies, mice were housed in sterilized micro-isolator cages; they received antibiotic-treated water (erythromycin/gentamycin) for the first 28 days after BMT and filtered water thereafter. 


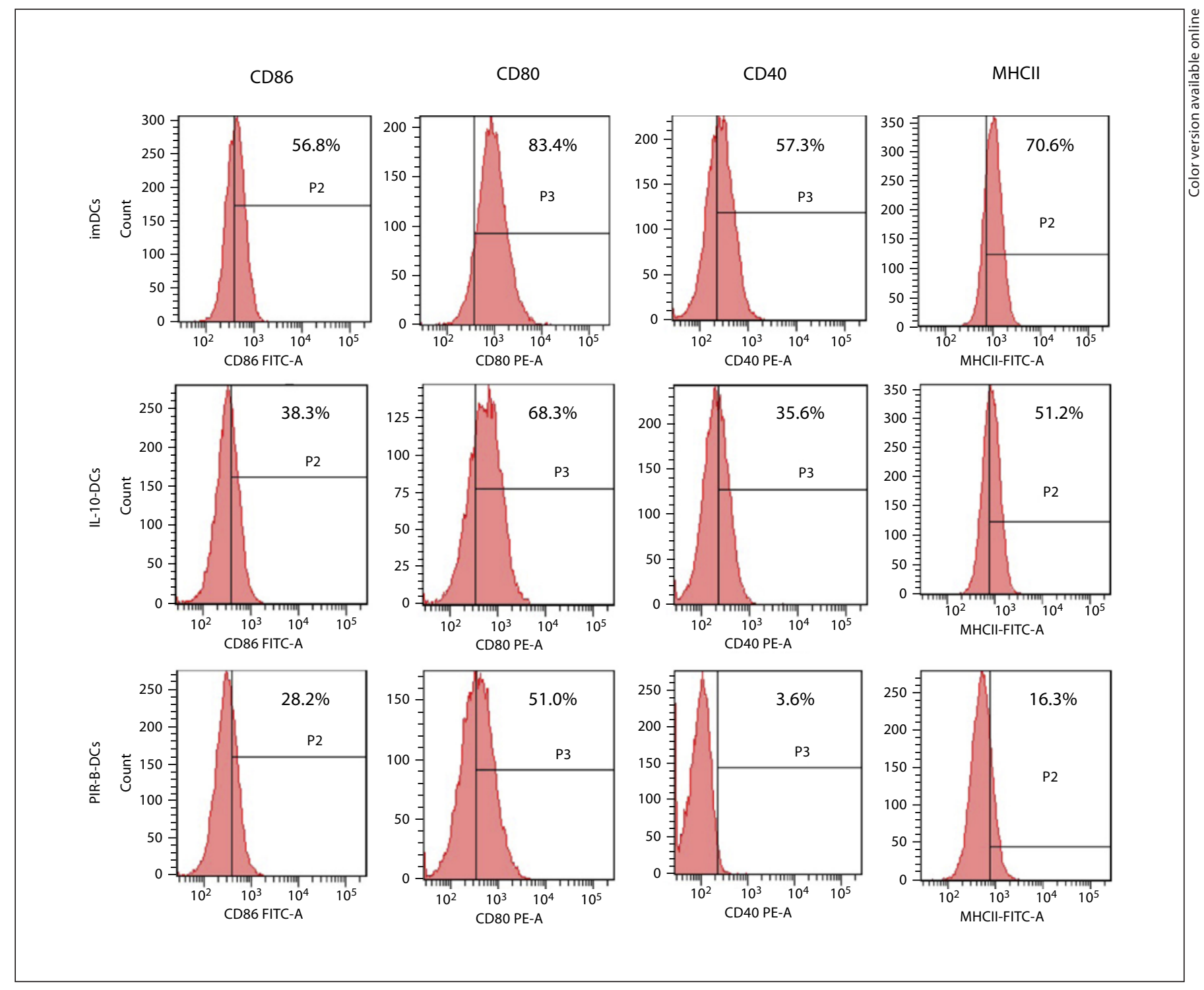

Fig. 1. Expressions of MHCII and CMs of DCs by FCM. Expression of CD86, CD80, CD40 and MHCII was determined in the three cell types by FCM analysis using proportional FITC-labeled anti-CD40, anti-CD80, antiCD86 or anti-MHCII antibodies ( $\mathrm{n}=3$, conducted in triplicate). In the top right corner of each graph, mean percentages of positive cells are given.

Systemic and Histopathologic Analysis of aGVHD

Survival and appearance were monitored daily until the mice died naturally of GVHD. The degree of clinical GVHD was assessed by a scoring system that sums changes in five clinical parameters: weight loss, posture, activity, fur texture and skin integrity (maximum index, 10) [10]. The index is more sensitive to aGVHD severity than weight loss alone, a parameter that has been found to be a reliable indicator of systemic GVHD in multiple murine models. For histology, moribund mice were euthanized to obtain the liver, intestines and skin, the primary GVHD target organs. Sections of tissue were fixed in $10 \%$ buffered formalin. Specimens were then embedded in paraffin and cut into $5-\mu \mathrm{m}-$ thick sections. Hematoxylin-eosin staining of paraffin-embedded tissue sections was performed according to standard protocols. Image acquisition was performed using an inverted phase contrast microscope (Olympus) with a SPOT RT camera and acquisition software.

\section{Statistical Analysis}

Differences were evaluated using a Statistical Package for Social Sciences (SPSS 14.0). Data were expressed as means and standard deviations. Student's paired t test or the log-rank test was performed to determine statistically significant differences. $\mathrm{p}<$ 0.05 was considered significant. 

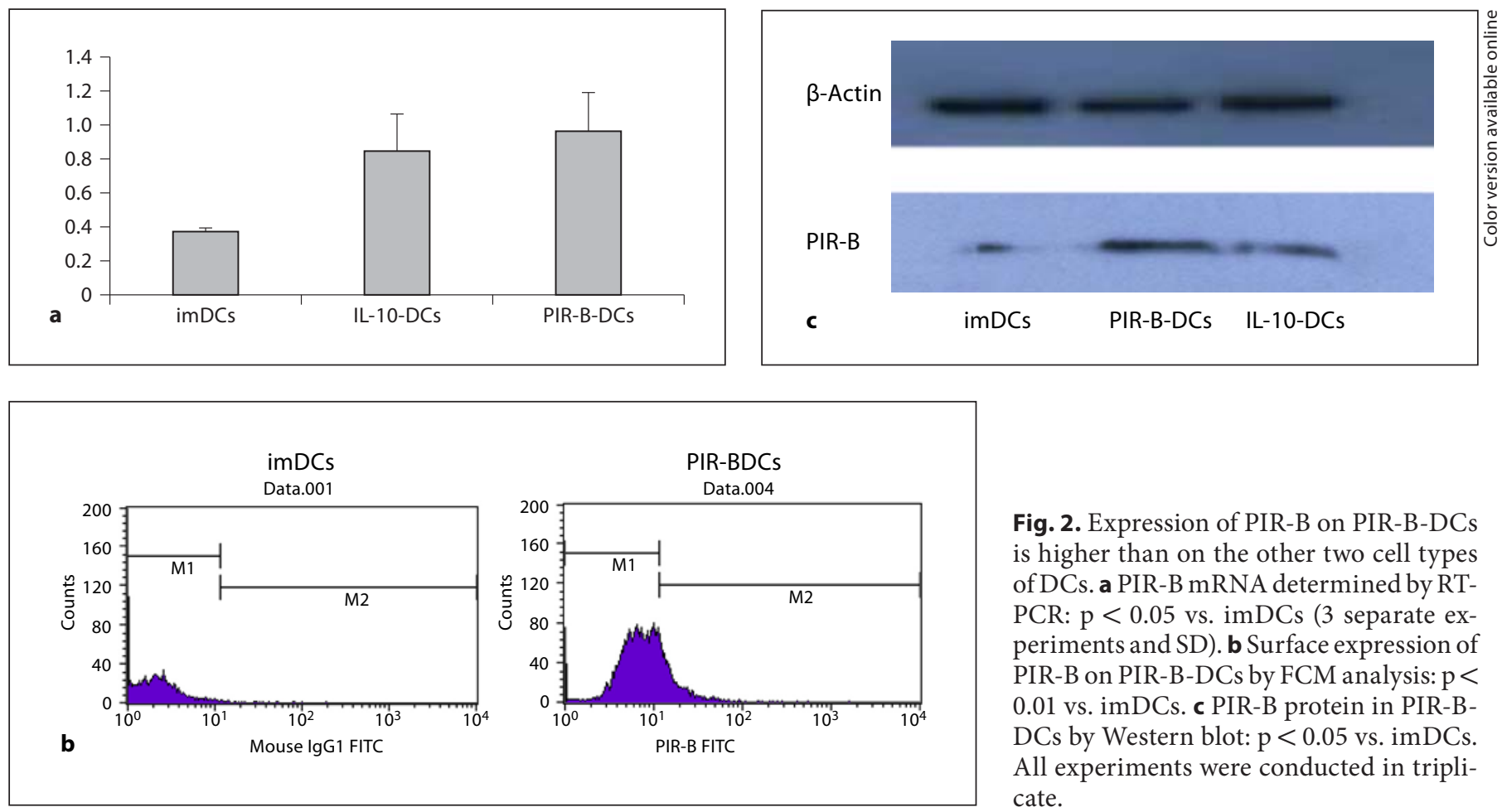

Fig. 2. Expression of PIR-B on PIR-B-DCs is higher than on the other two cell types of DCs. a PIR-B mRNA determined by RTPCR: $p<0.05$ vs. imDCs (3 separate experiments and SD). $\mathbf{b}$ Surface expression of PIR-B on PIR-B-DCs by FCM analysis: $\mathrm{p}<$ 0.01 vs. imDCs. c PIR-B protein in PIR-BDCs by Western blot: $\mathrm{p}<0.05$ vs. imDCs. All experiments were conducted in triplicate.

\section{Results}

PIR-B Lentivirus Inhibits the Expression of $M H C$ and CMs of BM-Derived DCs

To examine the effects of PIR-B expression on DCs, we stably transfected imDCs in a lentivirus expression vector for PIR-B. MHCII and CM expression was determined by flow cytometry (FCM). Compared to imDCs, both IL-10-DCs and PIR-B-DCs inhibited the expression of CD80, CD86, CD40 and MHCII, whereas PIR-B-DCs expressed lower levels of surface CMs (fig. 1). PIR-B-DCs showed a statistically significant decrease in MHC and CM expression (Student's paired t test, $\mathrm{p}<0.05$ ).

\section{Estimation of the Expression of PIR-B in DCs}

In a previous report, PIR-B played a crucial role in mediating murine-derived DC tolerance. Therefore, we analyzed the expression of PIR-B mRNA on imDCs, IL-10DCs and PIR-B-DCs by RT-PCR. Expression of PIR-B mRNA in PIR-B-DCs was stronger than that on IL-10DCs and imDCs (fig. 2a).

The expression of PIR-B on DCs was also estimated by FCM. Consistent with the results of RT-PCR, expression of PIR-B on imDCs, IL-10-DCs and PIR-B-DCs was 5.4 \pm 0.2 versus $14.1 \pm 1.22$ versus $23.6 \pm 2.16 \%(\mathrm{n}=3, \mathrm{p}<$
0.05). Mean fluorescence intensity of imDCs and PIR-BDCs amounted to $2.37 \pm 0.16$ versus $9.52 \pm 0.11(\mathrm{n}=3$, $\mathrm{p}<0.01$; fig. 2b). Moreover, PIR-B protein expression on imDCs, IL-10-DCs and PIR-B-DCs by Western blot was $0.327 \pm 0.078$ versus $0.807 \pm 0.256$ versus $0.955 \pm 0.218$ ( $\mathrm{n}=3, \mathrm{p}<0.05$; fig. $2 \mathrm{c}$ ).

Treatment of Hosts with Recipient-Derived PIR-B-DCs Reduced Death from a GVHD

The above results indicated that imDCs genetically modified to highly express PIR-B could efficiently exert a tolerogenic DC function. We therefore tested the in vivo suppressive function of murine DCs genetically modified to express PIR-B using a murine aGVHD model. Recipients were divided into four groups injected intravenously with imDCs, IL-10-DCs, PIR-B-DCs or no DCs from $\mathrm{BALB} / \mathrm{c}$ mice, respectively, at the time of BM-splenocyte transplantation. Controls that received only body TBI without BMT died within 10 days. In the BMT group, all $\mathrm{BALB} / \mathrm{c}$ recipients died on day 24 after transplantation of C57BL/6 BM and splenocytes. In these mice, clinical symptoms of aGVHD, such as reduced mobility, hair ruffling, weight loss and diarrhea, became apparent within 6 days. In contrast, $46.7 \%$ of BALB/c recipients of BMsplenocytes transplantation and PIR-B-DCs survived 


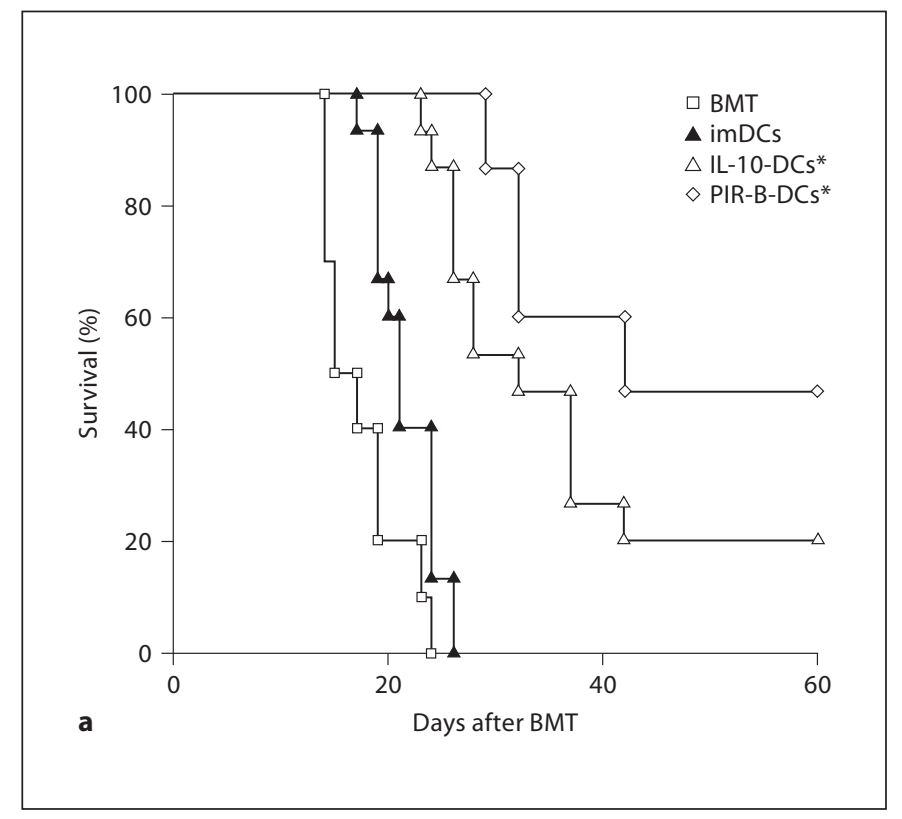

$>60$ days without apparent aGVHD, whereas only $20 \%$ of $\mathrm{BALB} / \mathrm{c}$ recipients of IL-10-DCs survived this long. An injection of imDCs at the time of transplantation of C57BL/6 BM and splenocytes to BALB/c recipients did not significantly affect aGVHD-induced lethality (fig. 3).

\section{Recipient-Derived PIR-B-DCs Protected Hosts against GVHD-Related Tissue Damage}

Our model was designed to study the effect of PIR-BDCs on aGVHD. Moribund mice with GVHD were euthanized and samples of the liver, skin, small intestine, kidney and spleen were taken for histopathologic analyses. In this BMT model, GVHD was histologically demonstrated as lymphocytic infiltration of the liver and skin (fig. 4). The small intestine, kidney and spleen were not affected (data not shown).

\section{Discussion}

DCs have emerged as a key component of the immune system involved in the induction of peripheral tolerance. Myeloid DCs are versatile APCs, which are derived from monocytes and hematopoietic BM cells [1]. In peripheral tissues, imDCs endocytose antigen, process it into peptides to form MHC-peptide complexes and migrate to lymph nodes where they present antigen to $\mathrm{T}$ cells [11]. However, when imDCs interact with $\mathrm{T}_{\mathrm{S}}$, they become

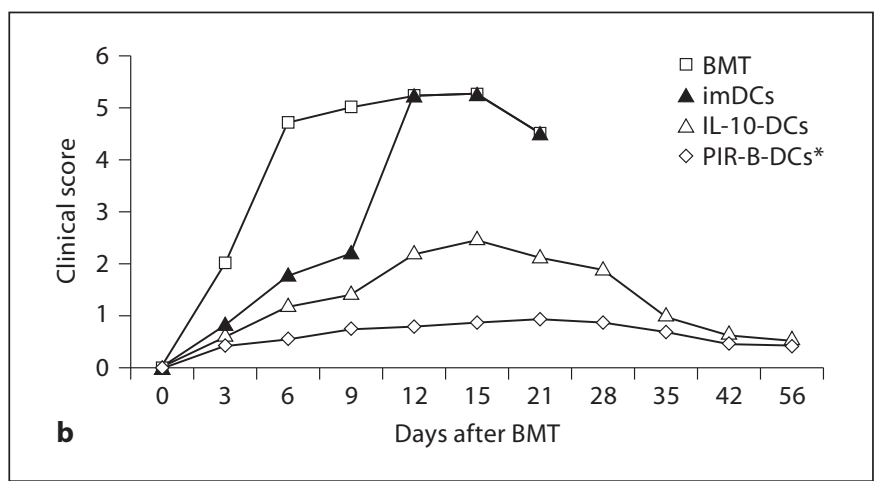

Fig. 3. Suppressive effect of PIR-B-DCs on aGVHD in murine allogeneic BMT. BALB/c mice that received C57BL/6 BMT were injected with imDCs, IL-10-DCs or PIR-B-DCs of BALB/c $\left(1 \times 10^{6} /\right.$ mouse, i.v.) or no DCs at the time of BMT. Mice were monitored daily for survival (a) and severity of symptoms (b). These results are representative of two individual experiments with similar results. log-rank test: ${ }^{*} \mathrm{p}<0.01$ vs. untreated mice that did not receive any DCs; t test: ${ }^{*} \mathrm{p}<0.01$.

tolerogenic and inhibit the activation of $\mathrm{T}_{\mathrm{H}}$ [2]. Expression profiling of tolerogenic DCs shows downregulation of the CMs CD40, CD80 and CD86 ligand and upregulation of the inhibitory receptors ILT2, ILT3 and ILT4 [12]. ILT2, ILT3 and ILT4 belong to a family of Ig-like inhibitory receptors that display a long cytoplasmic tail containing immunoreceptor tyrosine-based inhibitory motifs. Transduction of ILT3 and ILT4 in DC lines results in the generation of APCs, which completely block the proliferation of allospecific $\mathrm{T}_{\mathrm{H}}$. Upon interaction with tolerogenic DCs, allospecific CD4+ $\mathrm{T}_{\mathrm{H}}$ are converted into MHCII-specific $\mathrm{T}_{\mathrm{R}}$ cells that expressed CD25, CTLA-4, GITR, CD45RO and FOXP3. Tolerogenic DCs have been shown to induce the generation of alloantigen-specific $\mathrm{CD} 4+\mathrm{CD} 25+\mathrm{CD} 45 \mathrm{RO}+\mathrm{CTLA}-4+, \mathrm{FOXP} 3+\mathrm{T}_{\mathrm{R}}$ cells that resemble $\mathrm{CD} 4+\mathrm{CD} 25+$ naturally occurring $\mathrm{T}_{\mathrm{R}}$ cells [13] and induce the differentiation of CD8+CD28-FOXP3+ $\mathrm{T}_{S}$ cells. $\mathrm{T}_{S}$ and $\mathrm{T}_{\mathrm{R}}$ inhibit NF- $\mathrm{KB}$ activation and transcription of CMs in target APCs expressing the MHCI and MHCII antigens of the cells used for priming. Therefore, tolerogenic DCs are crucial to the generation of antigen-specific CD8+ $T_{S}$ and $C D 4+T_{R}$. The bidirectional interaction between regulatory cells and tolerogenic DCs perpetuates a cascade of events that downregulates T-cell alloreactivity. Tolerogenic DCs expressing the inhibitory receptors ILT3 and ILT4 induce immunosuppression by blocking the capacity of APCs to stimulate $\mathrm{CD} 4+\mathrm{T}_{\mathrm{H}}$ and CD8+ cytotoxic T cells. 

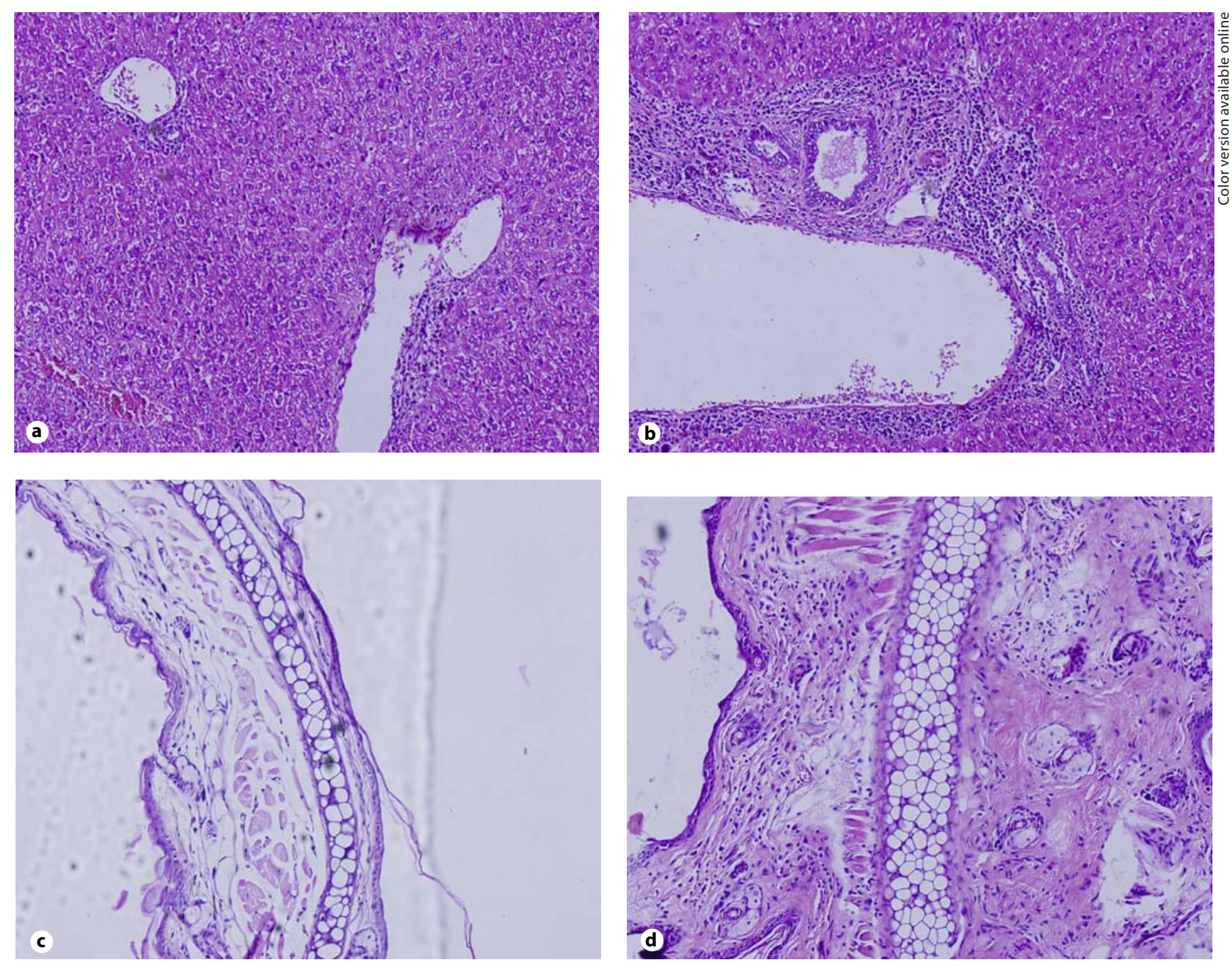

Fig. 4. Histopathologic analysis of recipient mice. Both liver (a) and skin (c) from mice injected with PIR-B-DCs showed slight lymphocytic infiltration, compared with significant lymphocytic infiltration of mice that only received BM-splenocyte transplantation $(\mathbf{b}, \mathbf{d})$.

Characteristically, the molecular structure and function of PIR-B expressed on APCs of rodents resembles ILT4 in humans. In this experiment, we transfected imDCs with PIR-B lentivirus and estimated PIR-B-DC tolerance. However, ILT4 ${ }^{\text {high }}$ tolerogenic DCs could be generated by treating myeloid DCs with IL-10 and IFN- $\gamma$ [3]. We used imDCs and IL-10-treated imDCs as controls to estimate the effects of treatment on DC tolerance. To define the molecular changes induced by different methods in DCs, MHC and CMs, DC expression was investigated by FCM analysis. We found that CD80, CD86, CD40 and MHCII expression was lower in PIR-B-DCs than in imDCs and IL-10-DCs. In other words, PIR-BDCs showed a stronger inhibitory effect on tolerogenic DCs. To estimate the expression of PIR-B on DCs, we analyzed mRNA protein of PIR-B on three DC types. Our data demonstrated that the expression of PIR-B on PIRB-DCs was significantly higher than that on imDCs and IL-10-DCs.

We also established a murine aGVHD model to investigate the in vivo suppressive function of murine DCs genetically modified to highly express PIR-B. However, in vivo analysis demonstrated that injection of PIR-B-DCs decreased morbidity and mortality during the process of 
aGVHD. In addition, GVHD-related tissue damage was less apparent in PIR-B-DC recipients. We presumed that injection of PIR-B-DCs could also induce a cascade of immunosuppressive events by activating the circulation of tolerogenic DCs and CD $8+\mathrm{T}_{\mathrm{S}} / \mathrm{CD} 4+\mathrm{T}_{\mathrm{R}}$. Patient $\mathrm{T}_{\mathrm{S}}$ has also been shown to induce upregulation of the inhibitory receptors ILT3 and ILT4 on donor APCs, as well as APCs transfected with a luciferase gene plasmid containing the ILT3 or ILT4 promoter $[14,15]$. Serial determination of suppressor activity displayed by patient CD4+CD25+ FOXP3 + T cells yielded similar results and showed that $\mathrm{T}_{\mathrm{R}}$-induced transcriptional activation of ILT3 and ILT4 in APCs matched to the donor [16].

In conclusion, PIR-B lentivirus-transfected DCs can efficiently induce specific suppressor function on alloreactive $\mathrm{T}$ cells and decrease mortality and morbidity correlated with GVHD.

\section{References}

1 Narita M, Takahashi M, Liu A, Ayres F, Satoh N, Abe T, Nikkuni K, Furukawa T, Toba K, Aizawa Y: Generation of dendritic cells from leukaemia cells of a patient with acute promyelocytic leukaemia by culture with GMCSF, IL-4 and TNF- $\alpha$. Acta Haematol 2001; 106:89-94.

$\checkmark 2$ Chang CC, Ciubotariu R, Manavalan JS, Yuan J, Colovai AI, Piazza F, Lederman S, Colonna M, Cortesini R, Dalla-Favera R, Suciu-Foca N: Tolerization of dendritic cells by T(S) cells: the crucial role of inhibitory receptors ILT3 and ILT4. Nat Immunol 2002;3: 237-243.

3 Manavalan JS, Rossi PC, Vlad G, Piazza F, Yarilina A, Cortesini R, Mancini D, SuciuFoca N: High expression of ILT3 and ILT4 is a general feature of tolerogenic dendritic cells. Transpl Immunol 2003;11:245-258.

$\checkmark 4$ Liu J, Liu Z, Witkowski P, Vlad G, Manavalan JS, Scotto L, Kim-Schulze S, Cortesini R, Hardy MA, Suciu-Foca N: Rat CD8+ FOXP3+ T suppressor cells mediate tolerance to allogeneic heart transplants, inducing PIR-B in APC and rendering the graft invulnerable to rejection. Transpl Immunol 2004;13:239-247.

5 Nakamura A, Kobayashi E, Takai T: Exacerbated graft-versus-host disease in Pirb-/mice. Nat Immunol 2004;5:623-629.
-6 Luo Y, Wang XC, Zou P: Construction and expression of PIR-B gene lentiviral vector. J Exp Hematol 2009;17:944-948.

7 Inaba K, Inaba M, Romani N, Aya H, Deguchi M, Ikehara S, Muramatsu S, Steinman RM: Generation of large numbers of dendritic cells from mouse bone marrow cultures supplemented with granulocyte/macrophage colony-stimulating factor. J Exp Med 1992;176:1693-1702.

8 Melhem NM, Liu XD, Boczkowski D, Gilboa E, Barratt-Boyes SM: Robust CD4+ and CD8+ T-cell responses to SIV using mRNAtransfected DC expressing autologous viral Ag. Eur J Immunol 2007;37:2164-2173.

$\checkmark 9$ Hoffmann P, Ermann J, Edinger M, Fathman CG, Strober S: Donor-type CD4(+)CD25(+) regulatory $\mathrm{T}$ cells suppress lethal acute graftversus-host disease after allogeneic bone marrow transplantation. J Exp Med 2002; 196:389-399.

$>10$ Cooke KR, Kobzik L, Martin TR, Brewer J, Delmonte J Jr, Crawford JM, Ferrara JL: An experimental model of idiopathic pneumonia syndrome after bone marrow transplantation: $\mathrm{I}$. The roles of minor $\mathrm{H}$ antigens and endotoxin. Blood 1996;88:3230-3239.

-11 Steinman RM, Nussenzweig MC: Avoiding horror autotoxicus: the importance of dendritic cells in peripheral $\mathrm{T}$ cell tolerance. Proc Natl Acad Sci USA 2002;99:351-358.
12 Suciu-Foca Cortesini N, Piazza F, Ho E, Ciubotariu R, LeMaoult J, Dalla-Favera R, Cortesini R: Distinct mRNA microarray profiles of tolerogenic dendritic cells. Hum Immunol 2001;62:1065.

13 Hori S, Nomura T, Sakaguchi S: Control of regulatory $\mathrm{T}$ cell development by the transcription factor Foxp3. Science 2003;299: 1057.

14 Chang CC, Ciubotariu R, Manavalan JS, Yuan J, Colovai AI, Piazza F, Lederman S, Colonna M, Cortesini R, Dalla-Favera R, Suciu-Foca N: Tolerization of dendritic cells by $\mathrm{T}(\mathrm{S})$ cells: the crucial role of inhibitory receptors ILT3 and ILT4. Nat Immunol 2002;3: 237.

15 Manavalan JS, Kim-Schulze S, Scotto L, Naiyer AJ, Vlad G, Colombo PC, Marboe C, Mancini D, Cortesini R, Suciu-Foca N: Alloantigen specific CD8+CD28- FOXP3+ T suppressor cells induce ILT3+ ILT4+ tolerogenic endothelial cells inhibiting alloreactivity. Int Immunol 2004;16:1055.

16 Suciu-Foca N, Manavalan JS, Scotto L, KimSchulze S, Galluzzo S, Naiyer AJ, Fan J, Vlad G, Cortesini R: Molecular characterization of allospecific T suppressor and tolerogenic dendritic cells. Int Immunopharmacol 2005; 5:7-11. 